\title{
Plasmonic Waveguide Modes of Film-Coupled Metallic Nanocubes
}

\author{
J. Britt Lassiter, ${ }^{*}, \dagger$ Felicia McGuire, ${ }^{\dagger, \ddagger}$ Jack J. Mock, $^{\dagger, \dagger}$ Cristian Ciraci, ${ }^{\dagger, \dagger}$ Ryan T. Hill, ${ }^{\S}$ \\ Benjamin J. Wiley," Ashutosh Chilkoti, ${ }^{\S}$ and David R. Smith ${ }^{*},+$, \\ ${ }^{\dagger}$ Department of Electrical and Computer Engineering, ${ }^{\ddagger}$ Center for Metamaterials and Integrated Plasmonics, ${ }^{\S}$ Department of \\ Biomedical Engineering, and "Department of Chemistry, Duke University, Durham, North Carolina 27708, United States
}

ABSTRACT: A metallic nanoparticle positioned over a metal film offers great advantages as a highly controllable system relevant for probing field-enhancement and other plasmonic effects. Because the size and shape of the gap between the nanoparticle and film can be controlled to subnanometer precision using relatively simple, bottom-up fabrication approaches, the film-coupled nanoparticle geometry has recently been applied to enhancing optical fields, accessing

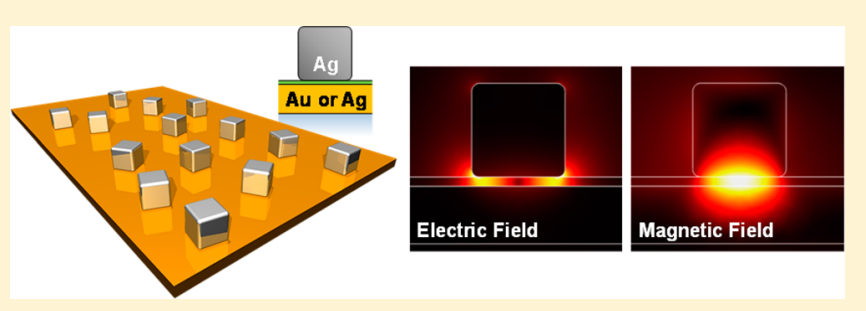
the quantum regime of plasmonics, and the design of surfaces with controlled reflectance. In the present work, we examine the plasmon modes associated with a silver nanocube positioned above a silver or gold film, separated by an organic, dielectric spacer layer. The film-coupled nanocube is of particular interest due to the formation of waveguide cavity-like modes between the nanocube and film. These modes impart distinctive scattering characteristics to the system that can be used in the creation of controlled reflectance surfaces and other applications. We perform both experimental spectroscopy and numerical simulations of individual nanocubes positioned over a metal film, finding excellent agreement between experiment and simulation. The waveguide mode description serves as a starting point to explain the optical properties observed.

KEYWORDS: Plasmon, perfect absorber, metasurface, nanocube, metal film, waveguide, transmission line, magnetic plasmon

$\mathrm{W}$ hen light impinges on a nanoscale metallic structure, resonant oscillations of the conduction electrons, known as surface plasmons, ${ }^{1,2}$ are excited. The unique properties of these surface plasmon resonances make them attractive for use in a wide array of applications. For example, when a plasmon is excited, it can induce large, highly localized electric fields, useful for enhancing molecular spectroscopies, ${ }^{3-5}$ refractive index sensing, 6,7 and nonlinear optics applications. ${ }^{8,9}$ For each of these applications, it is critical to employ a plasmonic nanostructure that can achieve a large enhancement of the optical fields, but it is also desirable to use nanostructures with easily tuned optical properties for precise deployment across a wide range of the electromagnetic spectrum. Simple, uncoupled nanoparticles cannot typically achieve these properties, yet when multiple plasmonic structures are coupled, qualities such as extreme field enhancements ${ }^{10,11}$ and broadly tunable resonances $^{12}$ become possible.

Coupled plasmonic nanostructures, such as nanoparticle dimers $^{12-15}$ or larger clusters of nanoparticles, ${ }^{16}$ have been studied extensively. Controlling and tuning the optical properties of these structures relies heavily on the ability to precisely manipulate the gap size between them, effectively determining how strongly they couple. As a result, much effort has been devoted toward fabrication techniques that guarantee control over this gap distance; yet, significant challenges remain. For example, top-down, lithographic fabrication techniques have excellent capabilities for placing and orienting complex structures but are typically unable to achieve controllable gap sizes smaller than $\sim 10 \mathrm{~nm}^{17-19}$ In contrast, the alternative approach of bottom-up, chemical synthesis can produce structures and composites with extremely small gap sizes but suffers from low yields and minimal control over position and orientation of the structures. ${ }^{20-23}$ Extremely precise manipulation of plasmonic junctions has also been achieved by using atomic force microscopy techniques to position two or more chemically fabricated particles in close proximity to each other. ${ }^{8,24-26}$ Similarly, electron beams have been shown to exert forces on nanoparticles, allowing for precise control over the junction between two nanoparticles. $^{27,28}$ While these techniques have resulted in unprecedented control and understanding of plasmonic junctions, they are limited to single nanostructures and cannot be used as a scalable fabrication technique. These issues have thus far prevented the fabrication of large-scale, practical devices using coupled plasmonic nanostructures.

The system of a metallic nanoparticle positioned over a metallic film ${ }^{29-36}$ represents an alternative class of coupled plasmonic structures that sidesteps the fabrication difficulties associated with the methods described above. In this geometry, nanoparticles can be fabricated by either chemical or lithographic approaches and subsequently directly deposited onto a metal film coated with a dielectric layer. The thickness of this dielectric layer can be precisely controlled by a variety of methods, facilitating manipulation of the gap between the particle and the film, and thus the plasmonic coupling. ${ }^{31,33}$ In fact, this geometry has recently been shown to be useful for

Received: July 18, 2013

Revised: October 14, 2013

Published: November 7, 2013 
ultraprecise measurement and application of enhanced electric fields. ${ }^{35,37}$ Furthermore, the gap size can be precisely maintained for many individual nanoparticles across a large sample, suggesting an immediate route toward the fabrication of large-scale, practical devices.

In the present work, we focus on the specific system of a chemically grown $\mathrm{Ag}$ nanocube $\mathrm{e}^{6,38-43}$ positioned over both $\mathrm{Ag}$ and Au films. This particular geometry, which can be thought of as an optical patch antenna supporting confined waveguide cavity modes between the metal layers, ${ }^{44-51}$ has recently been shown to possess a remarkable "perfect absorber" modality where the system behaves as a metasurface that is impedancematched to air, resulting in a theoretical zero reflection coefficient at the wavelength of operation. ${ }^{7,36,52-60}$ Because of the potential for a wide range of applications that leverage the unique properties of this system, it is worthwhile to examine the plasmon modes of film-coupled nanocubes in greater detail. Here we perform numerical analysis and experimental spectroscopy of $\mathrm{Ag}$ nanocubes over both $\mathrm{Ag}$ and $\mathrm{Au}$ films with a precisely sized molecular spacer layer defining the gap between them. We study this system as a function of gap size and determine the nature of its plasmon modes.

Silver nanocubes of various sizes were chemically synthesized using reaction conditions similar to previously reported syntheses. ${ }^{61}$ Five milliliters of ethylene glycol (EG) in a capped $50 \mathrm{~mL}$ round-bottom flask (rbf) were heated and stirred in a hot oil bath at $150{ }^{\circ} \mathrm{C}$ until equilibrated $(\sim 2 \mathrm{~min})$. Sixty microliters of $1.3 \mathrm{mM}$ sodium hydrosulfide $(\mathrm{NaSH})$ in $\mathrm{EG}$ were then pipetted into the rbf, which then remained capped in the oil bath. After $2 \mathrm{~min}, 500 \mu \mathrm{L}$ of $3 \mathrm{mM}$ hydrochloric acid $(\mathrm{HCl})$ in EG and $1.25 \mathrm{~mL}$ of poly(vinyl pyrrolidone) (PVP, $20 \mathrm{mg}$ / $\mathrm{mL}$ ) in EG were added, sequentially, and the rbf was once again capped. After a final two minutes, $400 \mu \mathrm{L} 0.0282 \mathrm{M}$ silver trifluoroacetate were added to the rbf. The flask was capped for a final time and allowed to react for 2-3 h, depending on the size of the nanocubes desired. Once the reaction was completed (solution was a deep tan color), the rbf was removed from the hot oil and placed in an ice bath to cool. The contents of the flask were transferred to a centrifuge tube, rinsed once with acetone to remove any remaining organics, and centrifuged at $5000 \mathrm{rpm}$ for $9 \mathrm{~min}$. The supernatant was removed, and the nanocube solution was resuspended in deionized water and vortexed briefly. The solution was then centrifuged a final time at $5000 \mathrm{rpm}$ for $4 \mathrm{~min}$, and the supernatant was again removed to produce the final solution. The addition of the PVP during the reaction results in a thin layer of PVP coating the nanocube, ${ }^{38}$ which we estimate to be $\sim 1-3 \mathrm{~nm}$.

Substrates were prepared by evaporating either a $50 \mathrm{~nm} \mathrm{Ag}$ film with a $4 \mathrm{~nm}$ Ge adhesion layer or a $50 \mathrm{~nm}$ Au film with a 5 $\mathrm{nm} \mathrm{Cr}$ adhesion layer onto a Nexterion Glass B Clean RoomCleaned glass slide via electron-beam evaporation. Dielectric spacer layers of varying thicknesses, composed of alternating polyelectrolyte (PE) layers of poly(allylamine hydrochloride) (PAH, MW $=\sim 58000)$ and polystyrene sulfonate (PSS, MW = $75000)$, were deposited onto the Ag film via layer-by-layer deposition. During each PE layer deposition step, the metal films were placed in solutions containing 0.003 mols-ofmonomer of the respective $\mathrm{PE}$ in $1 \mathrm{M} \mathrm{NaCl}$ for $5 \mathrm{~min}$ ( $\mathrm{Ag}$ films) or $30 \mathrm{~min}$ (Au films), rinsed thoroughly with ultrapure water, and then briefly rinsed in $1 \mathrm{M} \mathrm{NaCl}$ for 30s before either immersing in the next alternate PE solution or terminating the PE layer deposition. (Although the data presented below for nanocubes on $\mathrm{Au}$ films uses polyelectrolyte spacer layers made using a 30 min deposition time, samples were also made using a 5 min deposition time with similar results. The $5 \mathrm{~min}$ deposition time for the Ag films was chosen to avoid oxidation.) The sequential deposition of PAH and PSS PE layers was continued until the desired thickness was attained, with the terminating layer being cationic (PAH). After the final deposition step, the films were rinsed with ultrapure water and dried under a stream of nitrogen.

Silver nanocubes were immobilized onto the terminal PE layer (PAH) through momentary contact with a diluted solution of the nanocubes $(1: 100)$ to ensure a low nanocube surface coverage, thus allowing each individual nanoparticle to be characterized and mapped. The films were rinsed with ultrapure water and dried with nitrogen. The geometry of the final Ag nanocube-over-metal film system is shown in Figure $1 \mathrm{~A}$.

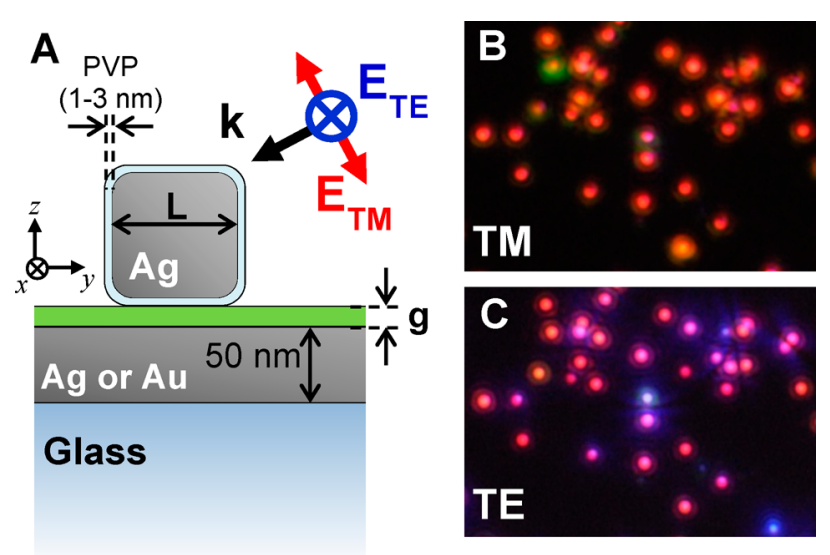

Figure 1. Ag nanocubes over a $\mathrm{Ag}$ or $\mathrm{Au}$ film. (A) Schematic representation of the geometry of the nanocube over metal film system. (B,C) Dark-field scattering images of $\mathrm{Ag}$ nanocubes over the metal film illuminated in the transverse magnetic polarization (B) and the transverse electric polarization (C). The TE and TM polarizatons are defined by the $\mathrm{E}$ vectors in panel $\mathrm{A}$.

Experimental scattering measurements were performed on individual nanocube structures via dark-field spectroscopy. Incoherent white light illumination was routed through a multimode optical fiber and focused onto the sample at an incidence angle of $62^{\circ}$ from the surface normal. This high angle of incidence in the dark-field configuration is necessary in order to ensure that the direct illumination, which is specularly reflected by the substrate, is not collected by the objective lens. As a result, only the light scattered by the nanoparticles is collected by the objective lens $(50 \times, \mathrm{NA}=0.55)$, and this scattered light can be either routed to a commercial, color digital camera (Nikon D70) for imaging or to a spectrometer/ CCD setup for spectral analysis. The illumination was polarized by a linear polarizer at the output of the fiber to be in either the tranverse electric (TE) or the transverse magnetic (TM) polarization (Figure 1A). Color images of a representative sample with the thinnest spacer layer are shown in Figure $1 \mathrm{~B}, \mathrm{C}$ for the two different polarizations. Under TM illumination, the particles appear red, while under TE illumination, the particles appear purple. Spectral analysis was performed by focusing an image of a particular particle onto an aperture in order to exclude all light that did not originate from the single particle under analysis, and then the spectrum of the particle was dispersed by a spectrograph (Acton 2300 SPI) and collected 
with a charge-coupled device (CCD) (CoolSnap EQ). The specific nanocubes used in the measurements were chosen by performing scattering spectroscopy of many individual nanoparticles and optically imaging their locations with respect to a large surface feature on the sample. Each measured nanoparticle was then located and imaged using a scanning electron microscope (SEM, FEI XL30 SEM-FEG). Clusters and misshapen particles were then excluded such that the data presented below represent only isolated nanocubes.

Numerical simulations of the scattering from film-coupled nanocubes were performed using the Finite Element Method (COMSOL Multiphysics). The simulated geometry was chosen to correspond as closely as possible with the experimental geometry. Plane wave illumination was incident at $62^{\circ}$ from the surface normal, and the scattered light was collected only in the solid angle above the nanoparticle corresponding to a 0.55 numerical aperture. In all cases, the simulations included a glass substrate $(n=1.47)$ underneath a $50 \mathrm{~nm}$ thick $\mathrm{Ag}$ or $\mathrm{Au}$ layer modeled using a dielectric function interpolated from reflectance and transmittance measurements on thin silver and gold films. ${ }^{62}$ A dielectric spacer layer $(n=1.4)$ of variable thickness was placed on top of the Ag layer in order to simulate the molecular spacer layer that defines the gap between the metal film and the nanocube. The Ag nanocube was positioned directly above this spacer layer, and the sizes of each of the nanocubes were taken directly from measurements in the SEM images of the samples (insets Figure 2). To more closely approximate the experimental geometry and to avoid numerical artifacts, the corners and edges of the nanocube were rounded to have a radius of $7 \mathrm{~nm}$ in all cases. The medium surrounding the nanocube above the substrate was modeled as air $(n=1)$. Finally, to better approximate the behavior of an infinitely extended metal film and avoid reflections due to truncating the simulation space, we used the scattered-field formulation in which the background field is specified using the analytical solution for the multilayered system (air-spacer-film-substrate) without the silver nanocube. ${ }^{63}$

A comparison of the representative experimental spectra and theoretical spectra for several individual nanocubes is shown in Figure 2. The column of panels on the left $(\mathrm{A}, \mathrm{C}, \mathrm{E})$ contains spectra of nanocubes over a silver film for three different gap spacer sizes (increasing from top to bottom), while the column of panels on the right $(\mathrm{B}, \mathrm{D}, \mathrm{E})$ contains spectra of nanocubes over a gold film, also for three different gap sizes (increasing from top to bottom). The size of the gap is determined by the number of PE spacer layers used. The spacers used here were made of $5(\mathrm{~A}, \mathrm{~B}), 7$ (C,D), and 9 (E,F) PE layers. The thicknesses of the PE gap spacers were measured by ellipsometry $^{64}$ (J. A. Woollam Co., inc., M-88 variable angle spectroscopic ellipsometer) for each of the six samples and were found to be (A) 5.8, (B) 6.4, (C) 10.9, (D) 11.6, (E) 11.4, and (F) $14.4 \mathrm{~nm}$. However, these nominal spacer thicknesses are effectively modified by the $\sim 1-3 \mathrm{~nm}$ PVP layer that surrounds the chemically fabricated nanocubes, resulting in actual gap sizes larger than the nominal spacer thickness. Because the plasmon modes of the nanocube-metal film system are mostly confined to the gap region, we do not include the PVP coating surrounding the entire nanocube in our simulations. Instead, we vary the spacer layer between the nanocube and the metal film in order to fit the experimental spectra. The spacer thickness was the only parameter varied in these simulations. For the nanocubes over the silver films, the thicknesses of the spacer layers used in the model were 8 (A),

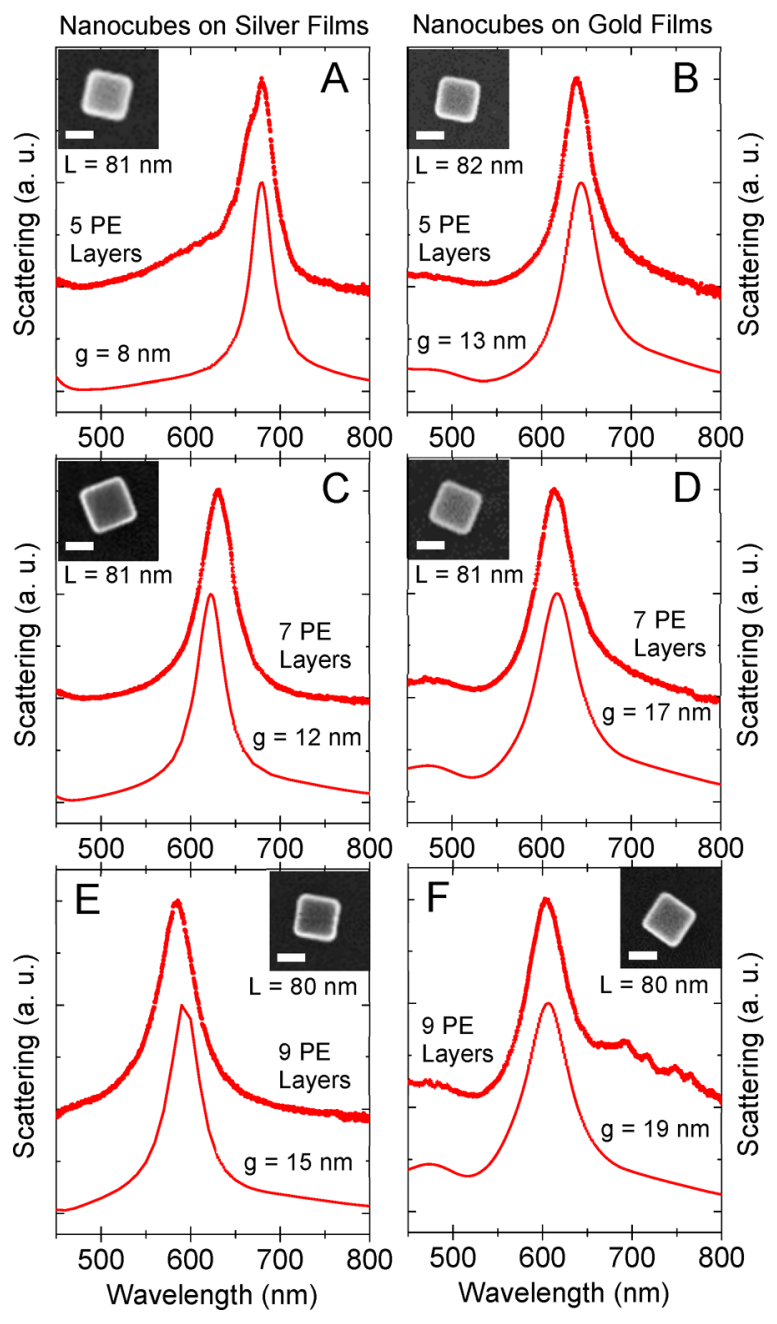

Figure 2. Experimental and simulated spectra for several individual nanocubes. Nanocubes over silver films are shown in the left column $(\mathrm{A}, \mathrm{C}, \mathrm{E})$ and nanocubes over gold films are shown in the right column $(B, D, F)$. In each panel, the experimental spectra are on the top and the simulated spectra are on the bottom. Insets show SEM images of the individual nanocube corresponding to the particular spectrum shown. All data was collected in the TM polarization as defined in Figure 1 (however, our simulations indicate that the resonance occurs at the same position in both the TE and TM polarizations). The length $(L)$ of the side of the nanocube is indicated for each nanocube. For both the silver and gold cases, three samples with different spacer layers made from $5(\mathrm{~A}, \mathrm{~B}), 7(\mathrm{C}, \mathrm{D})$, and $9(\mathrm{E}, \mathrm{F})$ polyelectrolyte layers are shown. The number of polyelectrolyte layers comprising the gap is indicated for each experimental spectrum, and the gap size is indicated for each simulated spectrum.

12 (C), and $15 \mathrm{~nm}(\mathrm{E})$. Each of these gap sizes is consistent with the measured PE layer thickness plus the 1-3 nm PVP layer on the nanocubes. For the nanocubes over gold films, the thicknesses of the spacer layer used in the model were 13 (B), 17 (D), and $19 \mathrm{~nm}$ (F). These fitted gap sizes are slightly larger than the expected values, but some variability between different batches of fabricated nanocubes is to be expected. Each of the spectra presented in Figure 2 represent nanocubes of similar size ( $\sim 80 \mathrm{~nm}$ side length). From these examples, one can observe that, as the gap size increases, the observed resonance shifts to shorter wavelengths for both silver and gold films. This behavior is expected for a coupled system where the coupling strength is determined by the proximity of the constituent 
structures, which in this case are the nanocube and the metal film.

In Figure 3, we show the full range of nanocube data that was collected in the experiments on both silver (A) and gold (B)
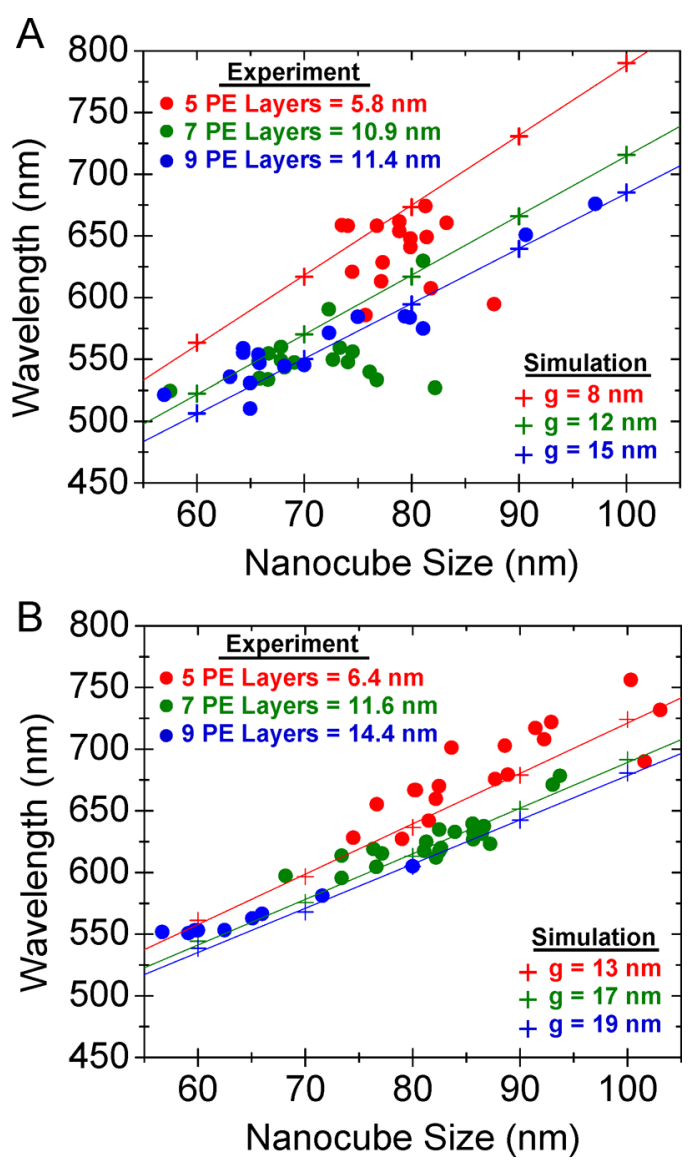

Figure 3. Nanocube plasmon resonance wavelength versus nanocube size: comparison of experimental data (circles) and simulations (crosses). The simulations use the same gap sizes from the fits in Figure 2. The lines represent linear fits to the simulated data. (A) Silver nanocubes on a silver film. (B) Silver nanocubes on a gold film.

films. Here, the peak wavelengths are shown as a function of the nanocube size as measured from the SEM images. The red, green, and blue circles represent the experimental data for the cases where the gap regions between the nanocube and the $\mathrm{Ag}$ film were spaced by 5, 7, and 9 PE layers, respectively. The red, green, and blue crosses depict the data points from numerical simulations where the gap sizes between the nanocube and the metal films were taken from the fits of the individual spectra in Figure 2, and the size of the nanocube was varied from 60 to $100 \mathrm{~nm}$. Linear fits to the simulated data points are shown as solid lines. The simulated data points show that the plasmon resonance of the nanocube redshifts both with decreasing gap size and with increasing nanocube size. However, in the case of silver films there exists a significant distribution in the experimentally measured data to the extent that this trend is not immediately obvious. Linear fits to the experimental data on silver films, where the slope of the fit line was held at the same value as the slope of the fit for the simulations, resulted in root-mean-square errors of $36.2,28.8$, and $16.8 \mathrm{~nm}$ for the 5,7 , and $9 \mathrm{PE}$ layer cases, respectively. However, for the case of gold films, the distribution of the experimental data appears to follow much more closely to the predictions from simulations. In this case, similar analysis resulted in root-mean-square errors of $19.9,12.2$, and $9.1 \mathrm{~nm}$ for the 5, 7, and 9 PE layer cases, respectively, representing a significant reduction in the error with respect to the silver films. We believe that the silver film is unstable over time and tends to oxidize, resulting in the irregular distribution of the experimental data in the silver film case.

The excellent agreement between our experiments and simulations confirms that our numerical model is adequately capturing the physics of the nanocube-metal film system. Thus, we can use the model to develop a greater understanding of the plasmon modes present in the system. We have chosen to further analyze the modes of the system (Figure 4) in the configuration from Figure 2A because this case had a small gap size, where the nanocube is strongly coupled to the metal film.
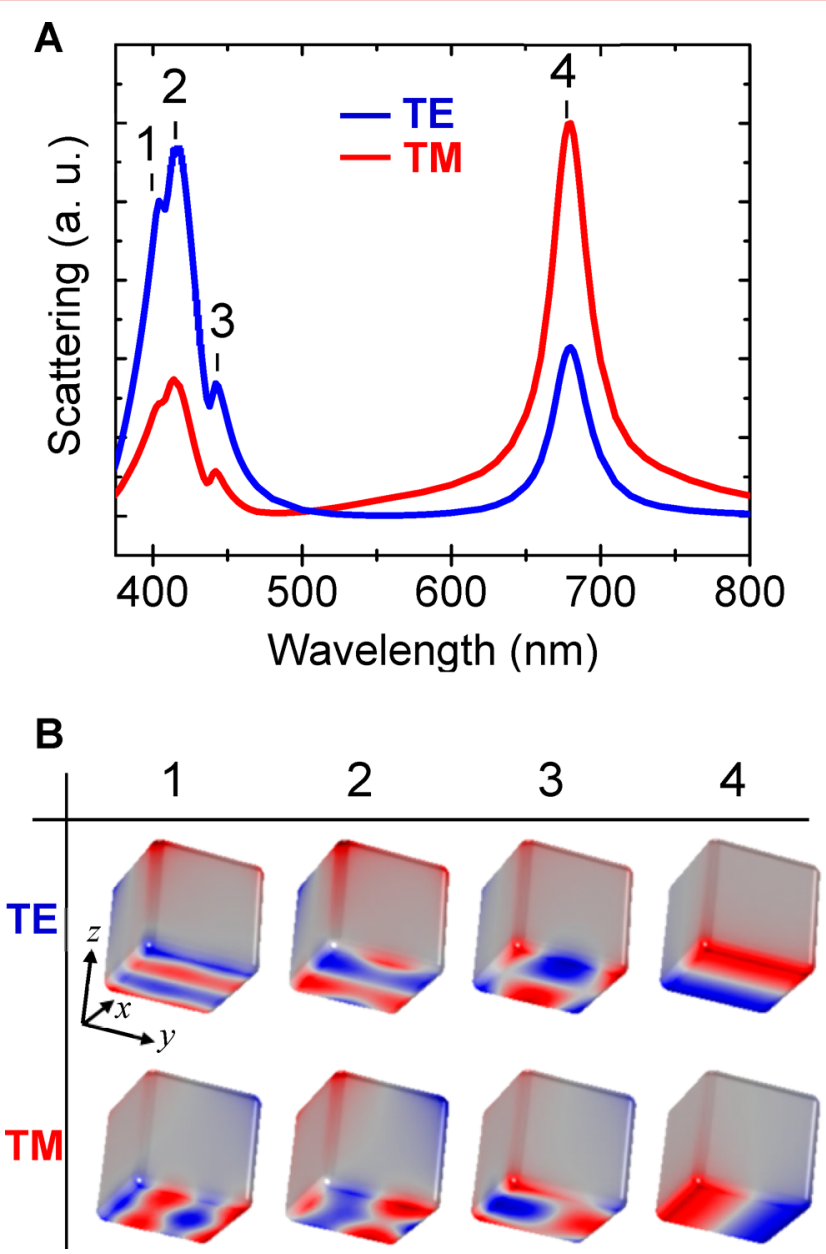

Figure 4. (A) Full range of simulated spectra from Figure $2 \mathrm{~A}$ (nanocube size $L=81 \mathrm{~nm}$, gap $g=8 \mathrm{~nm}$ ). (B) Electric displacement fields on the nanocube surface at each numerically indicated peak from panel A. Polarizations TE and TM were defined in Figure 1.

Our experimental measurements in Figure 2 were limited by the range of the detector such that any resonances occurring at wavelengths shorter than $\sim 450 \mathrm{~nm}$ were not observable. However, by extending the spectral range of our simulation, additional higher order modes in the blue region of the spectrum become apparent (Figure 4A, peaks 1, 2, and 3), while peak 4 represents the experimentally observable resonance. Here, we have simulated both the TM and the TE 
polarizations as defined in Figure 1. Note that each of these modes occurs at the same wavelength in both polarizations, but that peak 4 is more strongly excited in the TM polarization while the higher order resonances are more strongly excited by the TE polarization. This observation explains the color change observed in the dark-field images in Figure 1. Because the particles strongly scatter blue light under TE polarization but still scatter red light, they appear purple in the digital color images.

From the simulated fields, it is possible to further analyze and categorize the modes by visualizing the normal component of the electric displacement field on the surface of the nanocube (Figure $4 \mathrm{~b}$ ). Each of the modes can be understood as a waveguide cavity mode that has been observed in other metalinsulator-metal (MIM) geometries. ${ }^{36,65-71}$ The modes are supported by a MIM waveguide bounded by the nanocube on the top and the metal film on the bottom. Note that for each mode, the opposite polarity field is present on the metal film (not shown). These waveguide modes can be both excited and reflected multiple times at the edges of the nanocube, leading to resonances that can be observed in the scattering spectrum due to the leaky cavity that allows some of the radiation to escape and couple to free space. The higher order modes (peaks 1-3) are examples of waveguide modes and occur at shorter wavelengths such that the cavity is longer than a halfwavelength of the mode. Thus several nodes are evident, especially for peaks 1 and 2. Peak 3 appears to show both $x$ and $y$ propagating components leading to the complex spatial dependence observed. Notice that the modes appear to be nearly identical in both polarizations, other than some minor variations, showing that these modes occur independent of polarization.

At the experimentally observable resonance (peak 4), two areas of opposite polarity displacement field are separated across the bottom side of the nanocube. This distribution is consistent for both the TM and TE polarization. However, this mode is not an electric dipole mode because the opposite charge distribution is present on the metal film just below the nanocube (not shown). Instead, peak 4 can also be explained as a waveguide cavity mode that roughly satisfies the halfwavelength criteria. In particular, the metal-insulator-metal region supports a transmission line type of mode modified by the plasmonic dispersion of the metal. ${ }^{72}$ At the edges of the cube, there is a large effective impedance mismatch and most of the energy is reflected back under the cube. The effective boundary conditions at the cube edges for this mode are nearly those of a perfect magnetic conductor (PMC), though modified somewhat due to the plasmonic dispersion. The properties of the transmission line mode along with the approximation of the boundary conditions are enough to explain the various mode patterns that emerge from the numerical simulations. In particular, the waveguide description also aids in understanding the experimentally observed dependence of the resonance position on both the size of the nanocube and the size of the gap between the nanocube and the metal film, as seen in Figures 2 and 3. For increasing nanocube size, the resonance redshifts because the length of the cavity increases and will therefore support a resonance at a longer wavelength. Similarly, as the gap narrows, the effective refractive index of the waveguide mode increases, which effectively lengthens the cavity, again resulting in a resonance condition at a longer wavelength. ${ }^{36}$
The computed electric and magnetic field distributions corresponding to the lowest order waveguide resonance are shown in Figure 5. The electric field profile follows the pattern
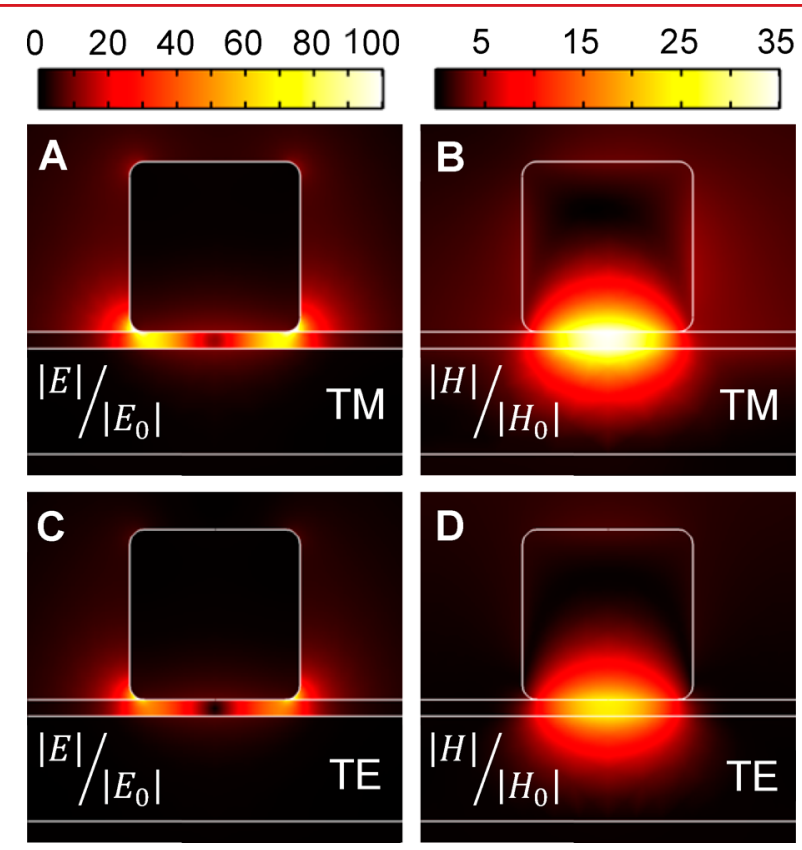

Figure 5. Enhancement of the electric $(A, C)$ and the magnetic $(B, D)$ fields for peak 4 from Figure 3. The field plots are shown for both the TM $(A, B)$ and the TE $(C, D)$ polarization. Frames $A$ and $C$ are plotted on the same scale for comparison (scale bar at the top for the electric field enhancement), and frames B and D are similarly plotted on the same scale (scale bar at the top for the magnetic field enhancement).

of the charge distribution on the bottom of the nanocube with two areas of intense field at either side of the nanocube. One of the more interesting aspects of this resonance is that the charge distribution essentially results in a current loop between the nanocube and metal film inducing a strongly enhanced magnetic field. This magnetic resonance has recently been used to create a "metasurface" that can perfectly absorb light. ${ }^{36}$

It is instructive to explore how the coupled modes of the nanocube-metal film system evolve into the uncoupled nanocube plasmon modes as the gap between the nanocube and the metal film is increased gradually (Figure 6). This progression of spectra was simulated in the TM polarization for both the case of a nanocube over a silver film (A) and a gold film (B). In these simulations, the dielectric spacer layer was removed for simplicity, such that the gap between the nanocube and the metal film was modeled as air $(n=1)$. For clarity, these spectra were normalized to the peak value of the $5 \mathrm{~nm}$ gap spectrum for both the gold and silver cases. This means that the relative amplitudes of the peaks can be compared among the silver film or the gold film case, but cannot be compared between the two cases. Note that this results in different peak amplitudes for the two air (black) spectra yet these are in fact the same spectrum. From this progression of spectra, one can see that, as the nanocube is moved away from the film, the magnetic waveguide mode strongly blueshifts because the coupling strength between the film and the nanocube is weakened with increasing distance. This trend is observed for both the silver film and gold film cases, although the mode is more redshifted in the gold film case. Similarly the higher order modes at $\sim 400 \mathrm{~nm}$ also weakly blueshift with increasing 


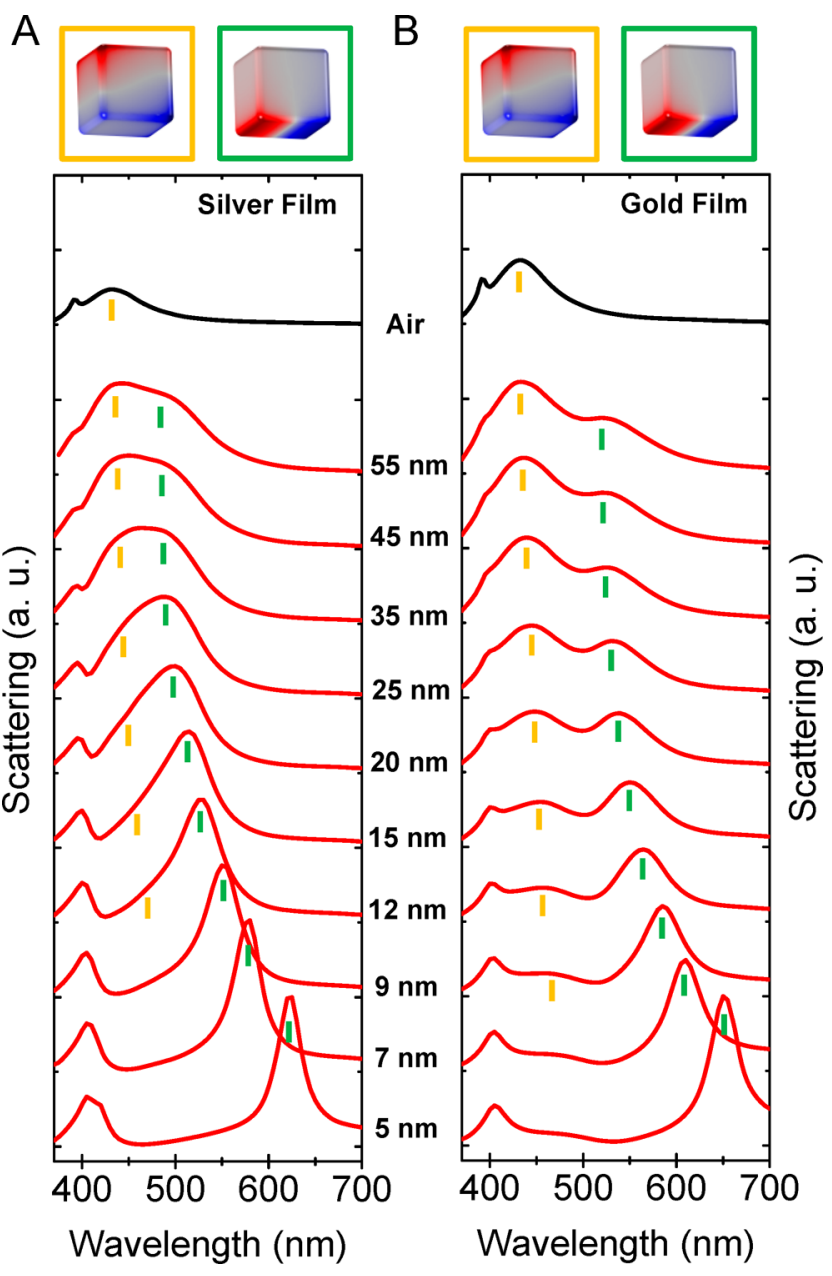

Figure 6. Simulated dependence of the scattering spectrum on the separation gap size, $g$, between an $81 \mathrm{~nm} \mathrm{Ag}$ nanocube and a $50 \mathrm{~nm}$ silver film (A) and a $50 \mathrm{~nm}$ gold film (B) for the TM polarizations. The gap sizes are indicated numerically next to each spectrum. The top spectrum in each polarization is labeled "air" to indicate that the nanocube was in a homogeneous air environment. (Top) Electric displacement field distributions for the two modes. Orange represents the dipolar mode in the "air" case and green represents the waveguide cavity mode in the $5 \mathrm{~nm}$ gap case.

distance from the metal film, although much less than the magnetic waveguide mode. Note that the magnetic waveguide mode (indicated by green markers) does not simply change gradually into the uncoupled dipole mode of the nanocube as the nanocube is moved away from the film. Instead, the magnetic waveguide mode begins to be less well-formed as the ability to couple to the metal film is removed at long separation distances. Simultaneously, the nanocube dipolar mode (indicated by orange markers) is damped out in close proximity to the metal film but begins to slowly emerge as the nanocube is moved away from the metal film. For the nanocubes over both the silver and gold films, this mode then slightly blueshifts to a final position of $440 \mathrm{~nm}$ and remains there when the metal film is completely removed and the nanocube is isolated in an isotropic air medium. The electric displacement field distributions are shown for both the dipolar mode (isolated in air case) and the waveguide mode ( $5 \mathrm{~nm}$ gap case). The dipolar nature of the dipolar mode (orange) is readily apparent and easily distinguishable from the waveguide mode (green).
In conclusion, we have fabricated and measured the spectra of individual Ag nanocubes coupled to silver and gold films. This choice of geometry coupled with the fabrication procedure using a molecular spacer layer to define the gap between the nanocube and the metal film provides a robust method to experimentally control a plasmonic junction with extreme precision. Using experimental and numerical analysis of this geometry, we found that the observed resonances can be understood as waveguide cavity modes, where the gap between the Ag nanocube and the metal film defines a waveguide cavity where electromagnetic energy can resonantly reflect back and forth. These waveguide modes are strongly dependent on the length of the waveguide, determined in this case by the size of the nanocube, offering a straightforward method for tuning the optical resonances over a broad range that in theory could be extended to span the visible and infrared frequencies. The waveguide resonances are also extremely sensitive to the gap size separating the nanocube from the metal film, a property that could potentially be exploited for active tuning applications.

\section{AUTHOR INFORMATION}

\section{Corresponding Authors}

*E-mail: (J. B. L.) britt.lassiter@duke.edu.

*E-mail: (D. R. S.) drsmith@ee.duke.edu.

\section{Notes}

The authors declare no competing financial interest.

\section{ACKNOWLEDGMENTS}

The authors would like to thank Dr. Stéphane Larouche for insightful discussions. This work was supported by the Air Force Office of Scientific Research under grants FA9550-09-10562 and FA9550-12-1-0491.

\section{REFERENCES}

(1) Lal, S.; Link, S.; Halas, N. J. Nat. Photonics 2007, 1, 641-648.

(2) Schuller, J. A.; Barnard, E. S.; Cai, W.; Jun, Y. C.; White, J. S.; Brongersma, M. L. Nat. Mater. 2010, 9, 193.

(3) Moskovits, M. Rev. Mod. Phys. 1985, 57, 783.

(4) Nie, S.; Emory, S. R. Science 1997, 275, 1102.

(5) Kneipp, K.; Wang, Y.; Kneipp, H.; Perelman, L. T.; Itzkan, I.; Dasari, R. R; Feld, M. S. Phys. Rev. Lett. 1997, 78, 1667.

(6) Sherry, L. J.; Chang, S.-H.; Schatz, G. C.; Duyne, R. P. V. Nano Lett. 2005, 5, 2034.

(7) Liu, N.; Mesch, M.; Weiss, T.; Hentschel, M.; Giessen, H. Nano Lett. 2010, 10, 2342.

(8) Danckwerts, M.; Novotny, L. Phys. Rev. Lett. 2007, 98, 026104.

(9) Kim, S.; Jin, J.; Kim, Y.-J.; Park, I.-Y.; Kim, Y.; Kim, S.-W. Nature 2008, 453, 757-760.

(10) Xu, H.; Aizpurua, J.; Kall, M.; Apell, P. Phys. Rev. E 2000, 62, 4318.

(11) Hao, E.; Schatz, G. C. J. Chem. Phys. 2004, 120, 357.

(12) Romero, I.; Aizpurua, J.; Bryant, G. W.; Abajo, F. J. G. d. Opt. Express 2006, 14, 9988.

(13) Sonnichsen, C.; Reinhard, B. M.; Liphardt, J.; Alivisatos, A. P. Nat. Biotechnol. 2005, 23, 741.

(14) Gunnarsson, L.; Rindzevicius, T.; Prikulis, J.; Kasemo, B.; Kall, M.; Zou, S. L.; Schatz, G. C. J. Phys. Chem. B 2005, 109, 1079.

(15) Kim, D.-S.; Heo, J.; Ahn, S.-H.; Han, S. W.; Yun, W. S.; Kim, Z. H. Nano Lett. 2009, 9, 3619.

(16) Fan, J. A.; Wu, C.; Bao, K.; Bao, J.; Bardhan, R.; Halas, N. J.; Manoharan, V. N.; Nordlander, P.; Shvets, G.; Capasso, F. Science 2010, 328, 1135 .

(17) Rechberger, W.; Hohenau, A.; Leitner, A.; Krenn, J. R.; Lamprecht, B.; Aussenegg, F. R. Opt. Commun. 2003, 220, 137. 
(18) Atay, T.; Song, J.-H.; Nurmikko, A. V. Nano Lett. 2004, 4, 1627.

(19) Jain, P. K.; Huang, W.; El-Sayed, M. A. Nano Lett. 2007, 7, 2080.

(20) Stoerzinger, K. A.; Hasan, W.; Lin, J. Y.; Robles, A.; Odom, T.

W. J. Phys. Chem. Lett. 2010, 1, 1046.

(21) Alivisatos, A. P.; Johnsson, K. P.; Peng, X.; Wilson, T. E.; Loweth, C. J.; M., P. B., Jr.; Schultz, P. G. Nature 1996, 382, 609.

(22) Mirkin, C. A.; Letsinger, R. L.; Mucic, R. C.; Storhoff, J. J. Nature 1996, 382, 607.

(23) Sardar, R.; Heap, T. B.; Shumaker-Parry, J. S. J. Am. Chem. Soc. 2007, 129, 5356.

(24) Olk, P.; Renger, J.; Hartling, T.; Wenzel, M. T.; Eng, L. M. Nano Lett. 2007, 7, 1736.

(25) Savage, K. J.; Hawkeye, M. M.; Esteban, R.; Borisov, A. G.; Aizpurua, J.; Baumberg, J. J. Nature 2012, 491, 574.

(26) Shafiei, F.; Monticone, F.; Le, K. Q.; Liu, X.-X.; Hartsfield, T.; Alu, A.; Li, X. Nat. Nanotechnol. 2013, 8, 95.

(27) Batson, P. E.; Reyes-Coronado, A.; Barrera, R. G.; Rivacoba, A.; Echenique, P. M.; Aizpurua, J. Nano Lett. 2011, 11, 3388.

(28) Scholl, J. A.; Garcia-Extarri, A.; Koh, A. L.; Dionne, J. A. Nano Lett. 2013, 13, 564.

(29) Leveque, G.; Martin, O. J. F. Opt. Lett. 2006, 31, 2750.

(30) Leveque, G.; Martin, O. J. F. Opt. Express 2006, 14, 9971.

(31) Mock, J. J.; Hill, R. T.; Degiron, A.; Zauscher, S.; Chilkoti, A.; Smith, D. R. Nano Lett. 2008, 8, 2245.

(32) Le, F.; Lwin, N. Z.; Steele, J. M.; Kall, M.; Halas, N. J.; Nordlander, P. Nano Lett. 2005, 5, 2009.

(33) Hu, M.; Ghoshal, A.; Marquez, M.; Kik, P. G. J. Phys. Chem. C 2010, 114, 7509.

(34) Lumdee, C.; Toroghi, S.; Kik, P. G. ACS Nano 2012, 6, 6301.

(35) Ciraci, C.; Hill, R. T.; Mock, J. J.; Urzhumov, Y.; FernandezDominguez, A. I.; Maier, S. A.; Pendry, J. B.; Chilkoti, A.; Smith, D. R. Science 2012, 337, 1072.

(36) Moreau, A.; Ciraci, C.; Mock, J. J.; Hill, R. T.; Wang, Q.; Wiley,

B. J.; Chilkoti, A.; Smith, D. R. Nature 2012, 492, 86.

(37) Moreau, A.; Ciraci, C.; Smith, D. R. Phys. Rev. B 2013, 87, 045401.

(38) Sun, Y.; Xia, Y. Science 2002, 298, 2176.

(39) Im, S. H.; Lee, Y. T.; Wiley, B.; Xia, Y. Angew. Chem., Int. Ed. 2005, 44, 2154.

(40) McLellan, J. M.; Li, Z.-Y.; Siekkinen, A. R.; Xia, Y. Nano Lett. 2007, 7, 1013.

(41) McMahon, J. M.; Wang, Y.; Sherry, L. J.; Duyne, R. P. V.; Marks, L. D.; Gray, S. K.; Schatz, G. C. J. Phys. Chem. C 2009, 113, 2731.

(42) Zhang, S.; Bao, K.; Halas, N. J.; Xu, H.; Nordlander, P. Nano Lett. 2011, 11, 1657.

(43) Gao, B.; Arya, G.; Tao, A. R. Nat. Nanotechnol. 2012, 7, 433.

(44) Jung, J.; Sondergaard, T.; Bozhevolnyi, S. I. Phys. Rev. B 2009, 79, 035401.

(45) Esteban, R.; Teperik, T. V.; Greffet, J. J. Phys. Rev. Lett. 2010, 104, 026802.

(46) Yousefi, L.; Foster, A. C. Opt. Express 2012, 20, 18326.

(47) Chakrabarty, A.; Wang, F.; Minkowski, F.; Sun, K.; Wei, Q.-H. Opt. Express 2012, 20, 11615.

(48) Yang, J.; Sauvan, C.; Jouanin, A.; Collin, S.; Pelouard, J.-L.; Lalanne, P. Opt. Express 2012, 20, 16880.

(49) Filter, R.; Qi, J.; Rockstuhl, C.; Lederer, F. Phys. Rev. B 2012, 85, 125429.

(50) Belacel, C.; Habert, B.; Bigourdan, F.; Marquier, F.; Hugonin, J-P.; Vasconcellos, S. M. d.; Lafosse, X.; Coolen, L.; Schwob, C.; Javaux, C.; Dubertret, B.; Greffet, J. J.; Senellart, P.; Maitre, A. Nano Lett. 2013, 13, 1516.

(51) Manolatou, C.; Rana, F. IEEE J. Quantum Electron. 2008, 44, 435.

(52) Landy, N. I.; Sajuyigbe, S.; Mock, J. J.; Smith, D. R.; Padilla, W. J. Phys. Rev. Lett. 2008, 100, 207402.

(53) Tao, H.; Bingham, C. M.; Strikwerda, A. C.; Pilon, D.; Shrekenhamer, D.; Landy, N. I.; Fan, K.; Zhang, X.; Padilla, W. J.; Averitt, R. D. Phys. Rev. B 2008, 78, 241103.
(54) Wen, Q.-Y.; Zhang, H.-W.; Xie, Y.-S.; Yang, Q.-H.; Liu, Y.-L. Appl. Phys. Lett. 2009, 95, 241111.

(55) Avitzour, Y.; Urzhumov, Y. A.; Shvets, G. Phys. Rev. B 2009, 79, 045131.

(56) Tao, H.; Bingham, C. M.; Pilon, D.; Fan, K.; Strikwerda, A. C.; Shrekenhamer, D.; Padilla, W. J.; Zhang, X.; Averitt, R. D. J. Phys. D: Appl. Phys. 2010, 43, 225102.

(57) Hao, J.; Wang, J.; Liu, X.; Padilla, W. J.; Zhou, L.; Qiu, M. Appl. Phys. Lett. 2010, 96, 251104.

(58) Wu, C.; B., N., III; Shvets, G.; John, J.; Milder, A.; Zollars, B.; Savoy, S. Phys. Rev. B 2011, 84, 075102.

(59) Tittl, A.; Mai, P.; Taubert, R.; Dregely, D.; Liu, N.; Giessen, H. Nano Lett. 2011, 11, 4366.

(60) Tuong, P. V.; Park, J. W.; Rhee, J. Y.; Kim, K. W.; Jang, W. H.; Cheong, H.; Lee, Y. P. Appl. Phys. Lett. 2013, 102, 081122.

(61) Zhang, Q.; Li, W.; Wen, L.-P.; Chen, J.; Xia, Y. Chem.-Eur. J. 2010, 16, 10234.

(62) Johnson, P. B.; Christy, R. W. Optical Constants of the Noble Metals. Phys. Rev. B 1972, 6, 4370.

(63) Hill, R. T.; Mock, J. J.; Urzhumov, Y.; Sebba, D. S.; Oldenburg, S. J.; Chen, S.-Y.; Lazarides, A. A.; Chilkoti, A.; Smith, D. R. Nano Lett. 2010, 10, 4150 .

(64) Hill, R. T.; Mock, J. J.; Hucknall, A.; Wolter, S. D.; Jokerst, N. M.; Smith, D. R.; Chilkoti, A. ACS Nano 2012, 6, 9237.

(65) Dionne, J. A.; Sweatlock, L. A.; Atwater, H. A.; Polman, A. Phys. Rev. B 2006, 73, 035407.

(66) Dionne, J. A.; Lezec, H. J.; Atwater, H. A. Nano Lett. 2006, 6, 1928.

(67) Gordon, R. Phys. Rev. B 2006, 73, 153405.

(68) Hill, M. T.; Marell, M.; Leong, E. S. P.; Smalbrugge, B.; Zhu, Y.; Sun, M.; Veldhoven, P. J. v.; Geluk, E. J.; Karouta, F.; Oei, Y.-S.; Nötzel, R.; Ning, C.-Z.; Smit, M. K. Opt. Express 2009, 17, 11107.

(69) Miyazaki, H. T.; Kurokawa, Y. Appl. Phys. Lett. 2006, 89, 211126.

(70) Bozhevolnyi, S. I.; Sondergaard, T. Opt. Express 2007, 15, 10869.

(71) Chandran, A.; Barnard, E. S.; White, J. S.; Brongersma, M. L. Phys. Rev. B 2012, 85, 085416.

(72) Ciraci, C.; Lassiter, J. B.; Moreau, A.; Smith, D. R. J. Appl. Phys. 2013, 114, 163108. 